\title{
The effect of cardiac rehabilitation on kinesiophobia in patients with coronary artery disease
}

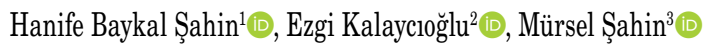 \\ ${ }^{1}$ Department of Physical Medicine and Rehabilitation, Karadeniz Technical University, School of Medicine, Trabzon, Turkey \\ ${ }^{2}$ Department of Cardiology, University of Health Sciences, Ahi Evren Chest and Cardiovascular Surgery Training and Research Hospital, Trabzon, Turkey \\ ${ }^{3}$ Department of Cardiology, Karadeniz Technical University, School of Medicine, Trabzon, Turkey
}

Received: August 16, 2019 Accepted: February 04, 2020 Published online: May 25, 2021

\begin{abstract}
Objectives: The aim of this study was to define the level of kinesiophobia in cardiac patients, to determine whether there was a positive change in kinesiophobia after an exercise based cardiac rehabilitation (CR), and to define the associated variables with the change in kinesiophobia.

Patients and methods: Between September 2017 and December 2018, a total of 98 patients (63 males, 35 females; mean age: $58 \pm 10.4$ years; range, 36 to 78 years) diagnosed with coronary artery disease (CAD) were included in the study. The Tampa Scale for Kinesiophobia Heart (TSK-SV Heart) was used to measure kinesiophobia. The short form of the International Physical Activity Questionnaire (IPAQ) was applied to measure physical activity level. The Short Form 36 (SF-36) was used to measure health-related quality of life (HRQoL). After CR, kinesiophobia was reevaluated.

Results: A high level of kinesiophobia was present in $74.5 \%$ of the patients. The mean TSK-SV heart score was $41.4 \pm 6.2$. After CR, $34.6 \%$ of the patients had a high level of kinesiophobia $(\mathrm{p}<0.001)$. The patients with a high level of kinesiophobia were physically more inactive than those with a low level of kinesiophobia $(\mathrm{p}=0.001)$ and HRQoL scores were significantly lower $(\mathrm{p}<0.05)$.

Conclusion: Kinesiophobia is quite common in patients with CAD. Aerobic exercise capacity and physical activity levels are lower in patients with a high level of kinesiophobia, compared to those with a low level of kinesiophobia. After an exercise-based CR program, kinesiophobia significantly reduces.
\end{abstract}

Keywords: Cardiac rehabilitation, coronary artery disease, kinesiophobia.

Cardiac rehabilitation (CR) is a comprehensive intervention including exercise training, risk factor education, behavior change, and psychological support. ${ }^{[1]}$ It is an essential part of cardiac patients' care. It slows or reverses disease progression by affecting the underlying risk factors and encourages a healthy and active lifestyle to improve quality of life, increase cardiac performance, increase exercise tolerance, reduce cardiovascular symptoms, decrease anxiety, depression and stress levels, return to work and maintain independence in daily living activities. ${ }^{[2]}$ Exercise-based CR is the intervention with the best scientific evidence to reduce mortality and morbidity in coronary artery disease (CAD), particularly after myocardial infarction, but also in chronic stable heart failure and cardiac surgery. ${ }^{[3]}$

Kinesiophobia is defined as excessive, irrational, and debilitating fear of movement and activity resulting from a feeling of vulnerability to painful injury or reinjury. ${ }^{[4]}$ In the literature, kinesiophobia have been observed in various medical conditions, such as chronic low back pain, osteoarthritis, fibromyalgia, and chronic musculoskeletal pain. ${ }^{[5-7]}$ Unfortunately,

Corresponding author: Hanife Baykal Şahin, MD. Karadeniz Teknik Üniversitesi Tıp Fakültesi Fiziksel Tip ve Rehabilitasyon Anabilim Dal, 61080 Trabzon, Türkiye. e-mail: baykalhanife@gmail.com

Baykal Şahin H, Kalaycıoğlu E, Şahin M. The effect of cardiac rehabilitation on kinesiophobia in patients with coronary artery disease. Turk J Phys Med Rehab 2021;67(2):203-210. 
there are few studies investigating the presence and effects of kinesiophobia in cardiac patients in the literature. A high level of kinesiophobia was found in $20 \%$ of patients with CAD, six months after the cardiac event. ${ }^{[8]}$ In another study, $86.7 \%$ of patients who underwent cardiac surgery had kinesiophobia preoperatively. ${ }^{[9]}$

The widely used questionnaire to measure kinesiophobia is the Tampa Scale for Kinesiophobia (TSK). ${ }^{[4]}$ The TSK was, then, revised again for heart diseases and TSK Swedish version for the Heart (TSK-SV Heart) was created. ${ }^{[10]}$ The TSK-SV Heart has been shown to be valid and reliable for heart diseases. ${ }^{[10]}$

Cardiovascular diseases are quite common and mortality rates are high. Physical inactivity is an important risk factor for these diseases. ${ }^{[1]}$ Therefore, it is conceivable that kinesiophobia may adversely affect these patients. In the present study, we aimed to define the level of kinesiophobia in CAD patients participating in a CR program, to determine whether there was a positive change in the level of kinesiophobia after an exercise-based CR program and to define associated variables with the changes in the level of kinesiophobia.

\section{PATIENTS AND METHODS}

This observational/cross-sectional study was conducted at Department of Cardiopulmonary Rehabilitation of Ahi Evren Chest and Cardiovascular Surgery Training and Research Hospital, between September 2017 and December 2018. A total of 98 patients (63 males, 35 females; mean age: $58 \pm 10.4$ years; range, 36 to 78 years) diagnosed with CAD were included in the study. The study included both newly diagnosed and previously diagnosed CAD patients. The patients were recruited from those who participated in an exercise-based CR program for the first time. Patients in the first month after acute myocardial infarction and patients with musculoskeletal or neurological disorders that would prevent exercise were excluded from the study. The study procedure was explained to the patients and a written informed consent was obtained from each participant. The study protocol was approved by Karadeniz Technical University School of Medicine Ethics Committee (date-no: 2018-151). The study was conducted in accordance with the principles of the Declaration of Helsinki.

Demographic data, medical history, cardiovascular risk factors and ejection fraction were recorded.
The TSK-SV Heart scale was used to evaluate kinesiophobia. The Turkish validity and reliability of the scale were conducted by Acar et al. ${ }^{[12]}$ in 2016 . The TSK-SV Heart consists of 17 questions which assess the subjective rating of kinesiophobia. Each item is rated on a four-point Likert-type scale as follows: 1-strongly disagree, 2-disagree, 3-agree, 4-strongly agree, and the total score varies between 17 and 68 . Higher values indicate a higher level of kinesiophobia. Values of $>37$ indicate kinesiophobia. ${ }^{[13]}$

The short form of the International Physical Activity Questionnaire (IPAQ) was applied to measure physical activity level over the seven-day period. It is a widely used reliable and valid tool for physical activity monitoring. ${ }^{[14]}$ The specific types of activity include walking, moderate-intensity activities, and vigorous-intensity activities. For all types, patients are asked to define on how many days and how many min they spent at each of them within the past seven days. The amount of metabolic equivalents (METs)-min is calculated by multiplying the amount of min with 8 (vigorous), 4 (moderate) or 3.3 (walking). The results are summed to gain an overall estimate of physical activity. The IPAQ form also divides individuals into three categories: 1-inactive, 2-minimally active, and 3 -active. The IPAQ has been shown to be valid and reliable in the Turkish population. ${ }^{[15]}$

The Short Form 36 (SF-36) was used to measure health-related quality of life (HRQoL). It is a widely used questionnaire measuring HRQoL through eight dimensions: physical functioning, role physical limitations, bodily pain, general health, vitality, social functioning, role emotional limitations, and mental health. ${ }^{[16]}$ The questions are combined to generate a score from 0 to 100 , with higher scores indicating a better health. The SF-36 is a useful scale for evaluating the patients with CAD. ${ }^{[17]}$

\section{Cardiac rehabilitation program}

The CR program was performed on the patients with a multidisciplinary team including a physiatrist as a medical director, a cardiologist, and an experienced physiotherapist. The main part of the rehabilitation program was aerobic exercise training. To determine exercise capacity, all patients underwent cardiopulmonary exercise test (CPET) on a cycle ergometer (Lode Corival, Groningen, the Netherlands). They were instructed to pedal (between 60 and $70 \mathrm{rpm}$ ), whilst workload was increased by 10 to $15 \mathrm{Watt} / \mathrm{min}$ depending on the expected physical fitness of the participant (based on sex, age, weight). Breath-by-breath gas analysis (Metalyzer ${ }^{\oplus} 3 \mathrm{~B}$; Cortex 
Medical, Leipzig, Germany) was recorded during exercise. The peak oxygen uptake $\left(\mathrm{VO}_{2}\right.$ peak) was defined as the highest value averaged over $30 \mathrm{sec}$ during the last stage of the incremental test and $\mathrm{VO}_{2}$ at the ventilatory threshold (VT) was determined by using the V-slope method. ${ }^{[18]}$ The peak oxygen uptake and oxygen uptake at the VT was also reported as a percentage of the predicted maximal oxygen consumption. ${ }^{[19]}$ Ventilatory efficiency was assessed by the slope of the min ventilation to the carbon dioxide production $\left(\mathrm{VE} / \mathrm{VCO}_{2}\right.$ slope) calculated over the linear phase of the response up to the VT. The maximal oxygen pulse (the ratio of $\mathrm{VO}_{2}$ to heart rate, reflects the amount of $\mathrm{VO}_{2}$ extracted per heart beat) was recorded. ${ }^{[19]}$ The same test was performed at the beginning and at the end of the CR program.

According to the CPET results, the aerobic exercise prescription was done individually. Exercise training was performed on a cycle ergometer. Each session lasted $30 \mathrm{~min}$, including a warm-up of 5 -min and a final 5 -min cool-down period at 25 to $40 \%$ of $\mathrm{VO}_{2}$ peak. The remaining $20 \mathrm{~min}$ of the exercise phase continued between 50 to $75 \%$ of $\mathrm{VO}_{2}$ peak. ${ }^{[20]}$ The Borg Scale of Rate of Perceived Exertion (RPE) was used; the patients exercised at an RPE of 13 to 15 . The load in the exercise phase was gradually increased by controlling the patient's heart rate and RPE throughout the six weeks. The patients continued

\begin{tabular}{|c|c|c|c|c|c|}
\hline \multicolumn{6}{|c|}{$\begin{array}{l}\text { TABLE } 1 \\
\text { Baseline demographic and clinical characteristics of patients }\end{array}$} \\
\hline Demographics & $\mathrm{n}$ & $\%$ & Mean \pm SD & Median & IQR \\
\hline Age (year) & & & $58.1 \pm 10.4$ & & \\
\hline \multicolumn{6}{|l|}{ Sex } \\
\hline Female & 35 & 35.7 & & & \\
\hline \multicolumn{6}{|l|}{ Admission diagnoses } \\
\hline Percutaneous coronary intervention & 69 & 70.4 & & & \\
\hline Coronary artery bypass grafting & 25 & 25.5 & & & \\
\hline Heart failure & 27 & 27.6 & & & \\
\hline Hypertension & 36 & 36.7 & & & \\
\hline Duration of illness (month) & & & $65.1 \pm 88.8$ & 24 & 106 \\
\hline Body mass index $\left(\mathrm{kg} / \mathrm{m}^{2}\right)$ & & & $29.7 \pm 5.8$ & & \\
\hline Former or current smoker & 33 & 33.7 & & & \\
\hline \multicolumn{6}{|l|}{ Clinical parameters } \\
\hline IPAQ score & & & $838.9 \pm 1212.5$ & 414.5 & 868 \\
\hline \multicolumn{6}{|l|}{ IPAQ subgroup } \\
\hline - Inactive & 66 & 67.3 & & & \\
\hline - Minimally active & 25 & 25.5 & & & \\
\hline - Active & 7 & 7.1 & & & \\
\hline SF-36 physical functioning & & & $54.3 \pm 24.7$ & & \\
\hline SF-36 role physical limitations & & & $37.3 \pm 38.3$ & 25 & 75 \\
\hline SF-36 role emotional limitations & & & $37.2 \pm 43.1$ & 0 & 100 \\
\hline SF-36 vitality & & & $43.8 \pm 23.6$ & & \\
\hline SF-36 mental health & & & $50.7 \pm 20.7$ & & \\
\hline SF-36 social functioning & & & $57.5 \pm 30.3$ & & \\
\hline SF-36 bodily pain & & & $60.1 \pm 27.2$ & & \\
\hline SF-36 general health & & & $49.8 \pm 22.1$ & & \\
\hline Tampa score & & & $41.4 \pm 6.2$ & & \\
\hline Tampa score $\geq 37$ & 73 & 74.5 & & & \\
\hline \multicolumn{6}{|l|}{ CPET parameters } \\
\hline $\mathrm{VO}_{2}$ peak $(\mathrm{mL} / \mathrm{kg} / \mathrm{min})$ & & & $14.6 \pm 3.0$ & & \\
\hline $\mathrm{VO}_{2}$-AT (\%) & & & $47.7 \pm 11.3$ & & \\
\hline $\mathrm{O}_{2}$ pulse $(\mathrm{mL})$ & & & $12.1 \pm 3.3$ & & \\
\hline Maximum power output (W) & & & $79.8 \pm 26.0$ & & \\
\hline $\mathrm{EQCO}_{2}$ & & & $32.5 \pm 3.6$ & & \\
\hline
\end{tabular}




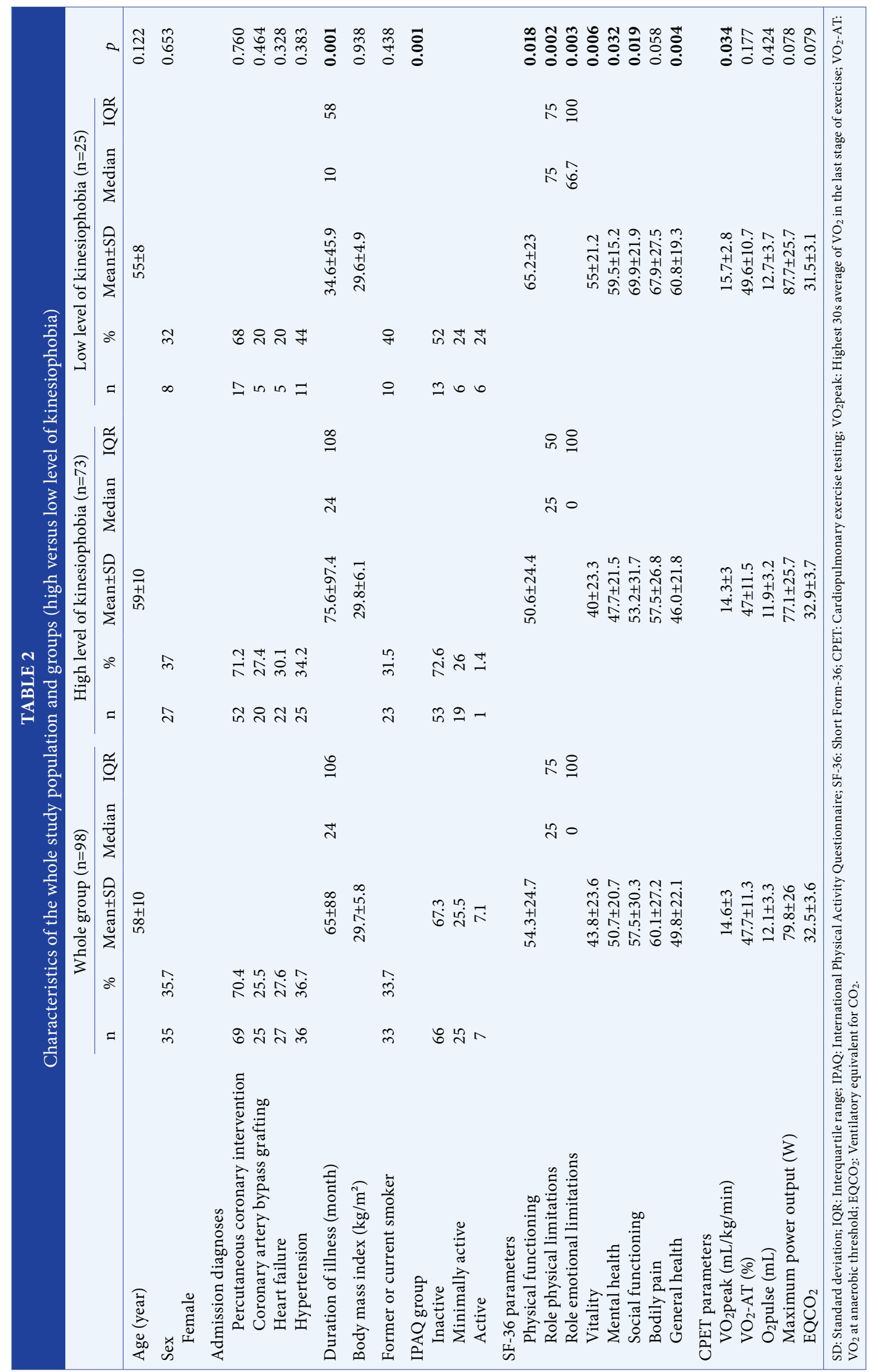


the CR program for five days a week, for a total of six weeks. Electrocardiograms were continuously tele-monitored and blood pressures were measured every $5 \mathrm{~min}$ throughout the exercise.

\section{Statistical analysis}

Statistical analysis was performed using the IBM SPSS version 23.0 software (IBM Corp., Armonk, NY, USA). Descriptive data were presented in mean \pm standard deviation (SD) or median (min-max) for continuous variables and in number and percentage for categorical variables. The chi-square test was used to analyze the differences between the ratios of categorical variables in independent groups and the McNemar test was used to compare dependent groups. The Kolmogorov-Smirnov test was used to evaluate the suitability of the data for normal distribution conditions. Comparisons of numerical variables between two independent groups were evaluated using the Student's t-test, when the normal distribution condition was satisfied and using the Mann-Whitney $\mathrm{U}$ test, when the normal distribution condition was not satisfied. Comparisons of numerical variables between two dependent groups were evaluated using the Wilcoxon test, since non-parametric conditions were provided. The Spearman correlation analysis was used to examine the relationship between the numerical variables between the two groups. The split-plot analysis of variance (ANOVA) was used to compare changes in numerical variables between two dependent groups. A $p$ value of $<0.05$ was considered statistically significant.

\section{RESULTS}

Baseline demographic and clinical characteristics of the patients are presented in Table 1. A high level of kinesiophobia was present in $74.5 \%$ of the patients. The mean duration of CAD was 65.1 (range, 1 to 240) months. The mean TSK-SV Heart score was $41.4 \pm 6.2$ (range, 27 to 60).

The differences between high versus low levels of kinesiophobia for demographic and clinical variables are presented in Table 2. There was no significant difference between the groups in terms of age, sex, body mass index, and smoking. The incidence of additional cardiac diagnoses, percutaneous coronary intervention (PCI), coronary artery bypass grafting

\begin{tabular}{|c|c|c|c|}
\hline \multicolumn{4}{|c|}{$\begin{array}{c}\text { TABLE } 3 \\
\text { Comparison of changes in CPET parameters after cardiac rehabilitation in patients with } \\
\text { a high versus low level of kinesiophobia }\end{array}$} \\
\hline & High level of kinesiophobia $(n=73)$ & Low level of kinesiophobia $(n=25)$ & \\
\hline & Mean \pm SD & Mean \pm SD & $p$ \\
\hline $\mathrm{VO}_{2}$ peak $(\mathrm{mL} / \mathrm{kg} / \mathrm{min})$ & & & 0.280 \\
\hline Baseline & $14.3 \pm 3$ & $15.7 \pm 2.8$ & \\
\hline Post-rehabilitation & $16 \pm 2.8$ & $17.9 \pm 2.7$ & \\
\hline$\Delta$ change & 1.7 & 1.9 & \\
\hline $\mathrm{VO}_{2}$-AT (\%) & & & 0.812 \\
\hline Baseline & $47 \pm 11.5$ & $49.6 \pm 10.7$ & \\
\hline Post-rehabilitation & $55.3 \pm 10.9$ & $57.5 \pm 13.1$ & \\
\hline$\Delta$ change & 8.4 & 7.4 & \\
\hline $\mathrm{O}_{2}$ pulse $(\mathrm{mL})$ & & & 0.332 \\
\hline Baseline & $11.9 \pm 3.2$ & $12.7 \pm 3.7$ & \\
\hline Post-rehabilitation & $12.8 \pm 2.8$ & $14 \pm 3.4$ & \\
\hline$\Delta$ change & 0.9 & 1.3 & \\
\hline Maximum power output (W) & & & 0.424 \\
\hline Baseline & $77.1 \pm 25.7$ & $87.7 \pm 25.7$ & \\
\hline Post-rehabilitation & $90.5 \pm 23.9$ & $103.4 \pm 23.8$ & \\
\hline$\Delta$ change & 13.6 & 15.3 & \\
\hline $\mathrm{EQCO}_{2}$ & & & 0.405 \\
\hline Baseline & $32.9 \pm 3.7$ & $31.5 \pm 3.1$ & \\
\hline Post-rehabilitation & $31.9 \pm 3.8$ & $30 \pm 2.3$ & \\
\hline$\Delta$ change & -0.9 & -1.6 & \\
\hline
\end{tabular}


(CABG), heart failure, and hypertension was similar in both groups. The duration of the disease was significantly longer in the group with a high level of kinesiophobia ( $\mathrm{p}=0.011)$. According to the IPAQ test, those with a high level of kinesiophobia were physically more inactive than those with a low level of kinesiophobia $(p<0.001)$. The HRQoL level measured with SF-36 was significantly lower for all subscales, except for bodily pain in patients with a high level of kinesiophobia ( $\mathrm{p}$ values for all subscales are shown in Table 2). When the CPET results were examined, the $\mathrm{VO}_{2}$ peak $(\mathrm{mL} / \mathrm{kg} / \mathrm{min})$ was significantly lower in the group with a high level of kinesiophobia $(\mathrm{p}=0.034)$.

The correlation between kinesiophobia level and demographic and clinical characteristics was analyzed. There was no significant correlation between age and kinesiophobia $(\mathrm{r}=0.188, \mathrm{p}=0.064)$. Kinesiophobia scores increased as the duration of the diagnosis increased, although the correlation was weak $(\mathrm{r}=0.262, \mathrm{p}=0.012)$. There was a significant decrease in the IPAQ scores with increasing kinesiophobia scores $(r=-0.315, p=0.002)$. All subscales of SF-36 were negatively correlated with the kinesiophobia scores $(\mathrm{p}<0.001)$. Kinesiophobia levels increased as peak $\mathrm{VO}_{2}$ level decreases; however, the correlation was weak $(\mathrm{r}=-0.256, \mathrm{p}=0.011)$.

All participants completed 30 sessions of CR. After CR, the patients were reevaluated in terms of kinesiophobia levels. High levels of kinesiophobia were detected in $74.5 \%$ of the patients before CR, and in $34.6 \%$ of the patients after CR. Initial TSK-SV Heart scores for the all population were $41.4 \pm 6.2$ and decreased to $34.9 \pm 5.7$ after CR $(\mathrm{p}<0.001)$. The mean TSK-SV Heart scores in the group with a high level of kinesiophobia were $43.6 \pm 4.9$ (range, 38 to 60 ) at baseline and $36.0 \pm 5.8$ (range, 25 to 51 ) after CR $(\mathrm{p}<0.001)$. The mean TSK-SV Heart scores in the group with a low level of kinesiophobia was $33.0 \pm 2$ (range, 27 to 37) at baseline and $30.9 \pm 3.6$ (range, 25 to 38) after CR $(\mathrm{p}=0.025)$.

Changes in kinesiophobia after CR were also compared in terms of demographic and clinical characteristics. There was a decrease in TSK-SV Heart scores after CR in both sexes; however, no significant difference was found between two sexes $(p=0.886)$. In addition, there was no significant difference between patients with and without additional cardiac diagnosis (PCI, CABG, heart failure and hypertension) in terms of change in TSK-SV Heart scores after CR. Following $\mathrm{CR}$, there were significant changes in the CPET parameters, compared to baseline $(\mathrm{p}<0.001$ for all); however, no significant difference was found between those with a high level of kinesiophobia and those with a low level of kinesiophobia (Table 3).

\section{DISCUSSION}

In the present study, we investigated the level of kinesiophobia in patients with CAD participating in a CR program and examined how CR would affect the level of kinesiophobia. Kinesiophobia was found in $74.5 \%$ of CAD patients. This rate was higher than the study on CAD patients six months after the cardiac event, in which $20 \%$ of the patients reported a high level of kinesiophobia. In the study of Brunetti et al., ${ }^{[21]}$ the rate of kinesiophobia was $86 \%$ in patients with acute coronary syndrome and $79 \%$ in patients with acute heart failure. These rates were similar to our study; however, we included both acute and chronic CAD patients in our study. We also found a positive correlation between kinesiophobia level and disease duration.

A study showed that the kinesiophobia level decreased over time after an acute CAD episode; however, a substantial part of the patients still had a high level of kinesiophobia across time. ${ }^{[22]}$ In the acute period after a cardiac event, it may be expected that patients will have a fear of activity. However, in our study, we found that kinesiophobia was also present in patients with long disease duration.

In the present study, those with a high level of kinesiophobia were physically more inactive than those with a low level of kinesiophobia. The $\mathrm{VO}_{2}$ peak value, which is an indicator of aerobic exercise capacity, was also significantly lower in patients with a high level of kinesiophobia than in those with a low level of kinesiophobia. A single study investigating the level of kinesiophobia and physical activity in patients with CAD was found in the literature and the results are consistent with our study: total IPAQ score was shown to be significantly lower in patients with a high level of kinesiophobia. ${ }^{[8]}$ Epidemiological studies have demonstrated that physical activity is effective in primary prevention of cardiovascular disease. Currently, it is known that physical activity plays a major role in secondary prevention and mortality reduction in patients with CAD. ${ }^{[23]}$ Kinesiophobia can be expected in heart patients. However, it would lead to prolonged physical inactivity, increased cardiac events, and mortality. Therefore, the immediate assessment and support of CAD patients in this perspective would contribute greatly to the long-term burden of disease. 
Furthermore, the HRQoL scores were found to be significantly lower in patients with a high level of kinesiophobia than those without kinesiophobia. Similar results are available in the literature. Bäck et al. ${ }^{[2]}$ found that HRQoL had a negative impact on kinesiophobia. In another study, fear of exercise was negatively associated with the HRQoL in patients with an internal cardiac defibrillator. ${ }^{[25]}$ Also, Kocjan and Knapik ${ }^{[26]}$ reported that poor self-assessment of health was associated with a greater intensification of kinesiophobia in patients undergoing CR. In the light of these results, we can speculate that kinesiophobia is closely related to quality of life. In this case, it is possible to suggest that psychotherapy for cardiac patients, which is an important part of CR, is indispensable in reducing the fear of activity and, thus, improving the quality of life.

In our study, the kinesiophobia level was reevaluated following $\mathrm{CR}$ and the rate decreased to $34.6 \%$. In other words, there was a significant improvement. Similarly, in one study, $86.6 \%$ of the patients undergoing cardiac surgery had kinesiophobia and this rate decreased to $6.6 \%$ after CR program and surgery. ${ }^{[9]}$ These data suggest that encouraging CAD patients to participate in a CR program would be an important step toward resolving the fear of activity in these patients. There are also studies indicating that the presence of kinesiophobia adversely affects participation in a CR program and its sustainability. ${ }^{[24,26]}$ Considering the fact that exercise-based CR reduces mortality and morbidity, encouraging patients to participate in a CR program is important for secondary prevention.

The TSK-SV Heart scale is not widely used in clinical practice. However, considering the high level of fear of exercise in patients with CAD, the implementation of a kinesiophobia scale while organizing the CR program would help to measure the outcome of rehabilitation. At the time of this study implementation, the TSK-SV Heart scale was the only available tool to evaluate fear of movement, although it was not specifically developed to measure kinesiophobia in CAD patients. Very recently, a novel scale developed for this purpose in CAD is published and, in future studies, this scale seems to be more appropriate for use in CAD. ${ }^{[27]}$

The main limitations of our study are its relatively small sample size and the lack of a limitation for the disease duration. Therefore, further large-scale studies with limited or grouped disease duration (early/late) would be beneficial. In addition, we evaluated kinesiophobia early after CR program in this study and further studies are needed to evaluate its long-term effect. The rate of angina pectoris or complications such as arrest, and intensive care hospitalization were not evaluated; however, these conditions may have contributed to fear of movement.

In conclusion, kinesiophobia is quite common in patients with CAD and increases, as the disease duration prolongs. Aerobic exercise capacity and physical activity levels are lower in patients with a high level of kinesiophobia, compared to those with a low level of kinesiophobia. In addition, the HRQoL decreases significantly. The CR can improve kinesiophobia significantly and this improvement seems to be higher in patients with a high level of kinesiophobia at baseline.

\section{Declaration of conflicting interests}

The authors declared no conflicts of interest with respect to the authorship and/or publication of this article.

Funding

The authors received no financial support for the research and/or authorship of this article.

\section{REFERENCES}

1. Anderson L, Thompson DR, Oldridge N, Zwisler AD, Rees $\mathrm{K}$, Martin N, et al. Exercise-based cardiac rehabilitation for coronary heart disease. Cochrane Database Syst Rev 2016;2016:CD001800.

2. Price KJ, Gordon BA, Bird SR, Benson AC. A review of guidelines for cardiac rehabilitation exercise programmes: Is there an international consensus? Eur J Prev Cardiol 2016;23:1715-33.

3. Piepoli MF, Corrà U, Benzer W, Bjarnason-Wehrens B, Dendale P, Gaita D, et al. Secondary prevention through cardiac rehabilitation: from knowledge to implementation. A position paper from the Cardiac Rehabilitation Section of the European Association of Cardiovascular Prevention and Rehabilitation. Eur J Cardiovasc Prev Rehabil 2010;17:1-17.

4. Kori S, Miller R, Todd D. Kinesiophobia: a new view of chronic pain behavior. Pain Manag 1990;3:35-43.

5. Altuğ F, Ünal A, Kilavuz G, Kavlak E, Çitişli V, Cavlak U. Investigation of the relationship between kinesiophobia, physical activity level and quality of life in patients with chronic low back pain1. J Back Musculoskelet Rehabil 2016;29:527-31.

6. Heuts PH, Vlaeyen JW, Roelofs J, de Bie RA, Aretz K, van Weel C, et al. Pain-related fear and daily functioning in patients with osteoarthritis. Pain 2004;110:228-35.

7. Roelofs J, Goubert L, Peters ML, Vlaeyen JW, Crombez G. The Tampa Scale for Kinesiophobia: further examination of psychometric properties in patients with chronic low back pain and fibromyalgia. Eur J Pain 2004;8:495-502. 
8. Bäck M, Cider Å, Herlitz J, Lundberg M, Jansson B. The impact on kinesiophobia (fear of movement) by clinical variables for patients with coronary artery disease. Int J Cardiol 2013;167:391-7.

9. Nair SP, Harania R, Rajguru V. Evaluation of Fear Avoidance Belief and Kinesiophobia in Patients after Undergoing InPatient Cardiac Rehabilitation Post Cardiac Surgery. International Journal of Health Sciences \& Research 2018;8:81-6.

10. Bäck M, Jansson B, Cider A, Herlitz J, Lundberg M. Validation of a questionnaire to detect kinesiophobia (fear of movement) in patients with coronary artery disease. J Rehabil Med 2012;44:363-9.

11. Benjamin EJ, Virani SS, Callaway CW, Chamberlain AM, Chang AR, Cheng S, et al. Heart Disease and Stroke Statistics-2018 Update: A Report From the American Heart Association. Circulation 2018;137:e67-e492.

12. Acar S, Savci S, Keskinoğlu P, Akdeniz B, Özpelit E, Özcan Kahraman B, et al. Tampa Scale of Kinesiophobia for Heart Turkish Version Study: cross-cultural adaptation, exploratory factor analysis, and reliability. J Pain Res 2016;9:445-51.

13. Vlaeyen JWS, Kole-Snijders AMJ, Boeren RGB, van Eek H. Fear of movement/(re)injury in chronic low back pain and its relation to behavioral performance. Pain 1995;62:363-72.

14. Craig CL, Marshall AL, Sjöström M, Bauman AE, Booth $\mathrm{ML}$, Ainsworth BE, et al. International physical activity questionnaire: 12 -country reliability and validity. Med Sci Sports Exerc 2003;35:1381-95.

15. Saglam M, Arikan H, Savci S, Inal-Ince D, Bosnak-Guclu $\mathrm{M}$, Karabulut E, et al. International physical activity questionnaire: reliability and validity of the Turkish version. Percept Mot Skills 2010;111:278-84.

16. Ware JE Jr. SF-36 health survey update. Spine (Phila $\mathrm{Pa}$ 1976) 2000;25:3130-9.

17. Failde I, Ramos I. Validity and reliability of the SF-36 Health Survey Questionnaire in patients with coronary artery disease. J Clin Epidemiol 2000;53:359-65.

18. Mezzani A, Hamm LF, Jones AM, McBride PE, Moholdt $\mathrm{T}$, Stone JA, et al. Aerobic exercise intensity assessment and prescription in cardiac rehabilitation: a joint position statement of the European Association for Cardiovascular Prevention and Rehabilitation, the American Association of Cardiovascular and Pulmonary Rehabilitation, and the Canadian Association of Cardiac Rehabilitation. J Cardiopulm Rehabil Prev 2012;32:327-50.

19. Balady GJ, Arena R, Sietsema K, Myers J, Coke L, Fletcher GF, et al. Clinician's Guide to cardiopulmonary exercise testing in adults: a scientific statement from the American Heart Association. Circulation 2010;122:191-225.

20. Lavie CJ, Thomas RJ, Squires RW, Allison TG, Milani RV. Exercise training and cardiac rehabilitation in primary and secondary prevention of coronary heart disease. Mayo Clin Proc 2009;84:373-83.

21. Brunetti ND, Guerra A, Ieva R, Correale M, Santoro F, Tarantino N, et al. Scared for the scar: fearsome impact of acute cardiovascular disease on perceived kinesiophobia (fear of movement). Clin Cardiol 2017;40:480-4.

22. Bäck M, Lundberg M, Cider Å, Herlitz J, Jansson B. Relevance of kinesiophobia in relation to changes over time among patients after an acute coronary artery disease event. J Cardiopulm Rehabil Prev 2018;38:224-30.

23. Winzer EB, Woitek F, Linke A. Physical activity in the prevention and treatment of coronary artery disease. J Am Heart Assoc 2018;7:e007725.

24. Bäck M, Cider Å, Herlitz J, Lundberg M, Jansson B. Kinesiophobia mediates the influences on attendance at exercise-based cardiac rehabilitation in patients with coronary artery disease. Physiother Theory Pract 2016;32:571-80.

25. van Ittersum $M$, de Greef $M$, van Gelder I, Coster J, Brügemann J, van der Schans C. Fear of exercise and health-related quality of life in patients with an implantable cardioverter defibrillator. Int J Rehabil Res 2003;26:117-22.

26. Kocjan J, Knapik A. Barriers of physical activity (kinesiophobia) in patients subjected to cardiac rehabilitation. Balt J Heal Phys Act 2014;6:291-7.

27. Ozyemisci-Taskiran O, Demirsoy N, Atan T, Yuksel S, Coskun O, Aytur YK, et al. Development and Validation of a Scale to Measure Fear of Activity in Patients With Coronary Artery Disease (Fact-CAD). Arch Phys Med Rehabil 2020;101:479-8.s 